Acta Crystallographica Section F

Structural Biology

and Crystallization

Communications

ISSN 1744-3091

Sen Liu, ${ }^{a}$ Guangteng Wu, ${ }^{\text {a }}$ Qichen Huang, ${ }^{a *}$ Luhua Lai, ${ }^{a}$ Youqi Tang, ${ }^{a}$ Hideaki Unno ${ }^{b}$ and Masami Kusunoki ${ }^{b}$

${ }^{\text {a }}$ State Key Laboratory for Structural Chemistry of Unstable and Stable Species, College of Chemistry, Peking University, Beijing 100871, People's Republic of China, and ${ }^{\mathbf{b}}$ Research Center for Structural Biology, Institute for Protein Research, Osaka University, 3-2 Yamada-oka, Suita, Osaka 565-0871, Japan

Correspondence e-mail: qchuang@chem.pku.edu.cn

Received 17 September 2004 Accepted 12 November 2004 Online 2 December 2004

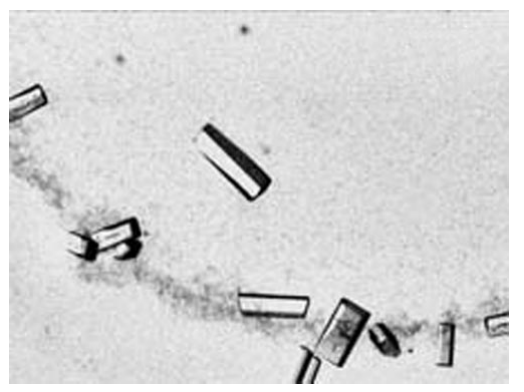

(C) 2005 International Union of Crystallography All rights reserved

\section{Expression, purification, crystallization and preliminary crystallographic study of a potential metal-dependent hydrolase with cyclase activity from Thermoanaerobacter tengcongensis}

The putative metal-dependent hydrolase gene TTE1006 from Thermoanaerobacter tengcongensis strain $\mathrm{MB}^{\mathrm{T}}$ ( $\mathrm{T}=$ type strain; Genbank accession No. AE008691) was heterologously expressed in Escherichia coli. The 205-aminoacid gene product was purified and crystallized. The crystal used for data collection belongs to space group $P 2_{1}$, with unit-cell parameters $a=85.2, b=62.1$, $c=172.4 \AA, \beta=104.2^{\circ}$. Using a synchrotron-radiation source, the resolution limit of the data reached $1.87 \AA$. Eight molecules were estimated to be present in the asymmetric unit, with a solvent content of $48 \%$. Structure determination is ongoing using the multiple-wavelength anomalous diffraction (MAD) method and also the molecular-replacement (MR) method.

\section{Introduction}

Thermoanaerobacter tengcongensis is an extremely thermophilic eubacterium that was isolated from Tengcong hot springs (Yunnan, China). Although T. tengcongensis shares several key features with the genus Thermoanaerobacter, it also displays some interesting phenotypic properties that are not identified with the genus (Xue et al., 2001; Fardeau et al., 2004; Acinas et al., 2004). To understand these contradictions and the molecular mechanisms by which this bacterium has adapted to extreme environments, the complete genomic sequence of $T$. tengcongensis as well as an analysis of its proteomic profile have recently been reported (Bao et al., 2002; Wang et al., 2004). Other papers have also referred to these mechanisms (Zhang et al., 2003; Sekowska et al., 2004; Irving et al., 2002).

We have cloned TTE1006, one of the genes from T. tengcongensis $\mathrm{MB}^{\mathrm{T}}$, and overexpressed it in Escherichia coli. The target protein TUH ( $T$. tengcongensis uncharacterized hydrolase) was predicted to be a metal-dependent hydrolase (Bao et al., 2002). Searching for homologous sequences in data banks using the BLAST program (NCBI; Marchler-Bauer et al., 2003) revealed that TUH has a putative conserved domain (COG1878) and is similar to some uncharacterized $\mathrm{Zn}^{2+}$-dependent polyketide cyclases (Hopwood, 1997). TUH has high sequence similarity with many ESTs (expressed sequence tags) from Homo sapiens, such as dbEST IDs 8439000 (sequence identity $25 \%$, similarity $41 \%$ ), 1713471 (sequence identity $25 \%$, similarity $42 \%$ ), 6101508 (sequence identity $27 \%$, similarity $45 \%$ ) and 3652714 (sequence identity $36 \%$, similarity $50 \%$ ) (TBLASTN; Altschul et al., 1997). A recently released $2.5 \AA$ resolution structure of a metal-dependent hydrolase from Bacillus stearothermophilus (PDB code 1r61; J. Maderova, D. Borek, D. Tomchick, A. Joachimiak, F. Collart \& Z. Otwinowski, unpublished work) has $55 \%$ sequence identity and $75 \%$ similarity to TUH (Fig. 1), which implies that TUH may adopt a similar structure. The structure determination of TUH to $1.87 \AA$ resolution will provide more detailed information for further functional studies.

\section{Expression and purification}

The TTE1006 gene was cloned into the expression vector pET-21a (Novagen) and TUH was expressed in E. coli strain BL21 (DE3) pLys (Novagen). The cells were suspended in $20 \mathrm{~m} M$ Tris- $\mathrm{HCl} \mathrm{pH} 7.6$, $10 \mathrm{~m} M$ 2-mercaptoethanol, $50 \mathrm{~m} M \mathrm{NaCl}, 1 \mathrm{~m} M$ PMSF, $1 \mathrm{~m} M \mathrm{ZnCl}_{2}$ 


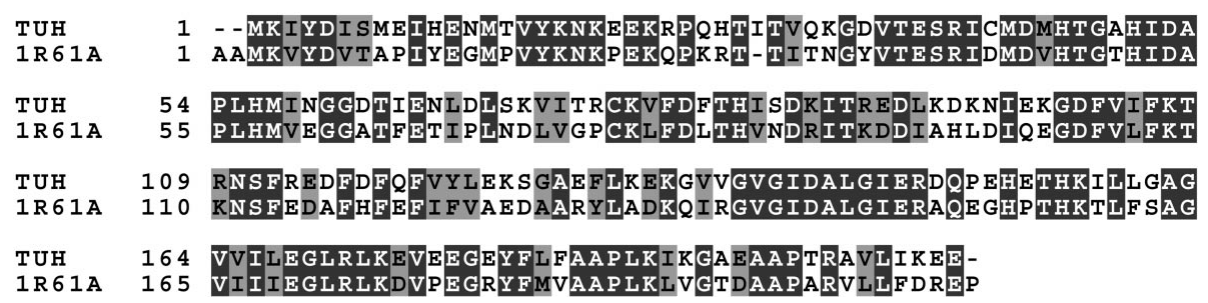

Figure 1

Sequence alignment of TUH and 1R61A calculated using ClustalX v.1.83 (Thompson et al., 1997). Identical amino acids are shown with black backgrounds and similar amino acids with grey backgrounds. 1R61A is the sequence of one of the two molecules in the asymmetric unit of the structure of a predicted metal-dependent hydrolase from B. stearothermophilus (PDB code 1r61).

and disrupted by sonication. The cell lysate was centrifuged (40 000g) for $30 \mathrm{~min}$ at $277 \mathrm{~K}$. The resulting supernatant was kept in a $338 \mathrm{~K}$ water bath for $10 \mathrm{~min}$ and then centrifuged $(40000 \mathrm{~g})$ for $30 \mathrm{~min}$ at $277 \mathrm{~K}$. The following procedures were all performed at room temperature. The supernatant was filtered with a $0.22 \mu \mathrm{m}$ filter and loaded onto a HiTrap Q HP column (5 ml; Amersham Biosciences) pre-equilibrated with buffer $A(20 \mathrm{~m} M$ bis-Tris- $\mathrm{HCl}$ pH 6.5, $10 \mathrm{~m} M$ 2-mercaptoethanol, $1 \mathrm{mM} \mathrm{ZnCl}_{2}$ ). Protein was eluted with a linear gradient of 0.0-1.0 $\mathrm{M} \mathrm{NaCl}$ in the same buffer. Fractions containing the target protein were pooled and further purified with a HiPrep 16/60 Sephacryl S-200 High-Resolution column (Amersham Biosciences). At each step, the target protein was analyzed by SDSPAGE with a $12 \%(w / v)$ acrylamide gel (Laemmli, 1970). SeMetsubstituted TUH was expressed in the auxotrophic E. coli strain B834 (DE3) (Novagen) and purified using a similar procedure.

\section{Crystallization}

TUH (wild type and SeMet-substituted) was crystallized by the hanging-drop vapour-diffusion method at $296 \mathrm{~K}$. The initial screening for the crystallization conditions was performed using the sparsematrix Crystal Screen I (Hampton Research). Small crystals appeared and the crystallization conditions were optimized. The initial protein concentration was $20 \mathrm{mg} \mathrm{ml}^{-1}$ in $50 \mathrm{mM}$ Tris- $\mathrm{HCl} \mathrm{pH}$ 7.6, $150 \mathrm{~m} M \mathrm{NaCl}, 20 \mathrm{~m} M$ 2-mercaptoethanol, $5 \mathrm{~m} M \mathrm{ZnCl}_{2}$, $0.02 \%(w / v) \mathrm{NaN}_{3}$. Drops were prepared by mixing $1 \mu \mathrm{l}$ protein solution with $1 \mu \mathrm{l}$ reservoir solution $[0.1 \mathrm{M}$ sodium citrate $\mathrm{pH} 5.8$, $20 \%(v / v)$ 2-propanol, $12 \%(w / v)$ PEG 4000] and were then equilibrated against $1 \mathrm{ml}$ of the same reservoir solution. Colourless rod-like crystals of WT and SeMet-substituted TUH were obtained after $9 \mathrm{~d}$ at $296 \mathrm{~K}$ (Fig. 2) with maximum dimensions of $0.10 \times 0.10 \times 0.70 \mathrm{~mm}$.

\section{Data collection and processing}

Crystals of both wild-type (WT) and SeMet-substituted TUH were exposed to X-ray radiation. Since the WT TUH did not diffract to a

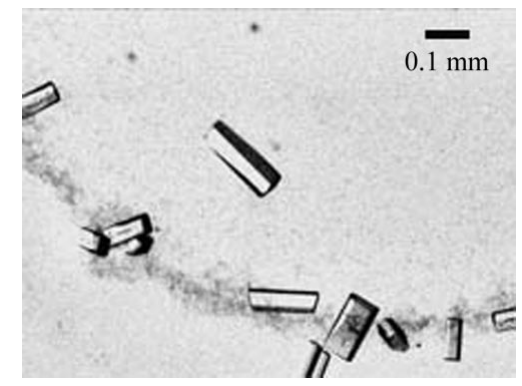

Figure 2

Crystals of SeMet-substituted TUH.
Table 1

Diffraction data statistics of the SeMet-substituted TUH.

R.m.s.d.s are given in parentheses; values in square brackets refer to the highest resolution shell.

\begin{tabular}{|c|c|c|c|}
\hline & High-energy remote & Edge & Peak \\
\hline X-ray source & \multicolumn{3}{|c|}{ Photon Factory, BL6A } \\
\hline Detector & \multicolumn{3}{|c|}{ ADSC Quantum, 4R CCD } \\
\hline Temperature (K) & \multicolumn{3}{|c|}{100} \\
\hline Space group & \multicolumn{3}{|l|}{$P 2_{1}$} \\
\hline \multicolumn{4}{|l|}{ Unit-cell parameters } \\
\hline$a(\AA)$ & $85.24(1)$ & $85.24(1)$ & $85.25(1)$ \\
\hline$b(\AA)$ & $62.06(2)$ & $62.06(1)$ & $62.07(2)$ \\
\hline$c(\AA)$ & $172.36(2)$ & $172.38(1)$ & $172.41(1)$ \\
\hline$\beta\left(^{\circ}\right)$ & $104.2(1)$ & $104.2(1)$ & $104.2(1)$ \\
\hline X-ray wavelengh $(\AA ̊)$ & 0.9000 & 0.97378 & 0.97885 \\
\hline Resolution range $(\AA)$ & $\begin{array}{l}50.00-1.87 \\
{[1.94-1.87]}\end{array}$ & $\begin{array}{l}50.00-1.87 \\
{[1.94-1.87]}\end{array}$ & $\begin{array}{l}50.00-1.87 \\
{[1.94-1.87]}\end{array}$ \\
\hline Total reflections & $514132[46726]$ & $472907[27103]$ & $924903[54409]$ \\
\hline Unique reflections & $142639[13743]$ & $138277[11293]$ & $139925[11828]$ \\
\hline Multiplicity & $3.6[3.4]$ & $3.4[2.4]$ & $6.6[4.6]$ \\
\hline$R_{\text {merge }} \dagger$ & $0.073[0.302]$ & $0.054[0.220]$ & $0.068[0.240]$ \\
\hline Completeness $(\%)$ & $98.5[95.1]$ & $95.4[69.7]$ & $95.7[73.0]$ \\
\hline Completeness $[I / \sigma(I)>2](\%)$ & $90.8[75.8]$ & $90.3[59.1]$ & $92.7[64.4]$ \\
\hline$\langle I / \sigma(I)\rangle$ & $15.6[3.4]$ & $19.9[4.2]$ & $26.9[5.2]$ \\
\hline$\Delta f^{\prime}$ & & -8.47 & -6.39 \\
\hline$\Delta f^{\prime \prime}$ & & 2.43 & 3.94 \\
\hline
\end{tabular}

$\dagger R_{\text {merge }}=\sum_{h k l} \sum_{i}\left|I(h k l)_{i}-\langle I(h k l)\rangle\right| / \sum_{h k l} I(h k l)$, where $I(h k l)_{i}$ is the $i$ th measurement of the intensity of reflection $h k l$ and $\langle I(h k l)\rangle$ is the mean intensity of reflection $h k l$.

resolution higher than $3 \AA$ (data not shown), only the data obtained for the SeMet-substituted protein have been processed and used.

Prior to data collection, a crystal of TUH was soaked in reservoir solution with the addition of $15 \%(w / v)$ PEG 4000 for $30 \mathrm{~h}$ and was then transferred to a nylon CryoLoop (Hampton Research) and dipped into liquid nitrogen prior to placement in a nitrogen-gas stream at $100 \mathrm{~K}$. Data were collected at beamline BL6A of the Photon Factory at the High Energy Acceleration Research Organization, Tsukuba, Japan using an ADSC Quantum 4R CCD camera (Watanabe et al., 1995). The values of $\Delta f^{\prime}$ and $\Delta f^{\prime \prime}$ were determined experimentally with X-ray absorption fine structure (XAFS) spectroscopy after the mounting of the crystal on the beamline. Three wavelengths were chosen to collect data separately. The oscillation ranges were $180^{\circ}$ for the edge and high-energy remote and $360^{\circ}$ for the peak wavelengths. Wavelengths were used in the sequence highenergy remote, edge and peak, with the oscillation per frame being $1^{\circ}$ in each case. The data were processed using the program HKL2000 (Otwinowski \& Minor, 1997). Diffraction data statistics are summarized in Table 1. The crystals belonged to the monoclinic space group $P 2_{1}$. Assuming the presence of eight molecules of TUH in the asymmetric unit, the value of the Matthews coefficient $V_{\mathrm{M}}$ is $2.37 \AA^{3} \mathrm{Da}^{-1}$, corresponding to a solvent content of $48 \%$, both of which are within the normal range of values for protein crystals (Matthews, 1968). $6 \times 8=48$ selenium positions have been determined by MAD and the structure determination of TUH is currently 
in progress using the MAD and MR methods, using the structure of a predicted metal-dependent hydrolase from B. stearothermophilus (PDB code 1r61) as a search model.

We thank Professor Runseng Chen of the Institute of Biophysics Academia Sinica for providing the T. tengcongensis $M B 4^{T}$ genomic DNA and help with target selection and Drs Soichi Wakatsuki and Noriyuki Igarash for kind help with data collection at the Photon Factory. This work was supported by grants from the 'Structural Genomics' section of the High Technology Development Program of China and the '863 Project of China' to QH and partially by Grantsin-Aid (Nos. 10558109 and 12480181) from the Japan Society for the Promotion of Science to MK.

\section{References}

Acinas, S. G., Marcelino, L. A., Klepac-Ceraj, V. \& Polz, M. F. (2004). J. Bacteriol. 186, 2629-2635.

Altschul, S. F., Madden, T. L., Schäffer, A. A., Zhang, J.-H., Zhang, Z., Miller,

W. \& Lipman, D. J. (1997). Nucleic Acids Res. 25, 3389-3402.

Bao, Q.-Y. et al. (2002). Genome Res. 12, 689-700.
Fardeau, M.-L., Salinas, M. B., L'Haridon, S., Jeanthon, C., Verhé, F., Cayol, J.-L., Patel, B. K. C., Garcia, J.-L. \& Ollivier, B. (2004). Int. J. Syst. Evol. Microbiol. 54, 467-474.

Hopwood, D. A. (1997). Chem. Rev. 97, 2465-2497.

Irving, J. A., Steenbakkers, P. J. M., Lesk, A. M., Op den Camp, H. J. M., Pike, R. N. \& Whisstock, J. C. (2002). Mol. Biol. Evol. 19, 1881-1890.

Laemmli, U. K. (1970). Nature (London), 227, 680-685.

Marchler-Bauer, A. et al. (2003). Nucleic Acids Res. 31, 383-387.

Matthews, B. W. (1968). J. Mol. Biol. 33, 491-493.

Otwinowski, Z. \& Minor, W. (1997). Methods Enzymol. 276, 307-326.

Sekowska, A., Dénervaud, V., Ashida, H., Michoud, K., Haas, D., Yokota, A. \& Danchin, A. (2004). BMC Microbiol. 4, 9.

Thompson, J. D., Gibson, T. J., Plewniak, F., Jeanmougin, F. \& Higgins, D. G. (1997). Nucleic Acids Res. 25, 4876-4882.

Wang, J.-Q., Xue, Y.-F., Feng, X.-L., Li, X.-L., Wang, H., Li, W., Zhao, C.-F., Cheng, X.-J., Ma, Y.-H., Zhou, P.-J., Yin, J. N., Bhatnagar, A., Wang, R. \& Liu, S.-Q. (2004). Proteomics, 4, 136-150.

Watanabe, N., Nakagawa, A., Adachi, S. \& Sakabe, N. (1995). Rev. Sci. Instrum. 66, 1824-1826.

Xue, Y.-F., Xu, Y., Liu, Y., Ma, Y.-H. \& Zhou, P.-J. (2001). Int. J. Syst. Evol. Microbiol. 51, 1335-1341.

Zhang, J., Liu, J.-F., Zhou, J., Ren, Y.-Y., Dai, X.-Y. \& Xiang, H. (2003). Biotechnol. Lett. 25, 1463-1467. 